\title{
Four-frequency solution in a magnetohydrodynamic Couette flow as a consequence of azimuthal symmetry breaking
}

\author{
F. Garcia, M. Seilmayer, A. Giesecke, and F. Stefani
}

(Dated: December 30, 2020)

\begin{abstract}
The occurrence of magnetohydrodynamic (MHD) quasiperiodic flows with four fundamental frequencies in differentially rotating spherical geometry is understood in terms of a sequence of bifurcations breaking the azimuthal symmetry of the flow as the applied magnetic field strength is varied. These flows originate from unstable periodic and quasiperiodic states with broken equatorial symmetry but having four-fold azimuthal symmetry. A posterior bifurcation gives rise to two-fold symmetric quasiperiodic states, with three fundamental frequencies, and a further bifurcation to a four-frequency quasiperiodic state which has lost all the spatial symmetries. This bifurcation scenario may be favoured when differential rotation is increased and periodic flows with $m$-fold azimuthal symmetry, $m$ being product of several prime numbers, emerge at sufficiently large magnetic field.
\end{abstract}

Understanding how systems become chaotic is of fundamental importance in many applications. Biological systems [1, 2], financial models [3], road traffic modelling [4], laser physics [5], neural networks [6], and simulations in fluid dynamics [7], or magnetohydrodynamics [8], exhibit transitions from regular oscillatory behaviour to a chaotic regime. Quite often, this transition follows the Newhouse-Ruelle-Takens (NRT) [9] scenario in which after a few bifurcations, involving quasiperiodic states, chaos emerges. According to the NRT theorem quasiperiodic oscillatory motions, which are known as tori, with 3 or more fundamental frequencies are unstable to small perturbations and thus unlikely to occur. However, the numerical experiments of [10] evidenced that the mathematical notion of small perturbations is a key issue and that in case of an appropriate spatial structure of the perturbations a three-tori solution may well be observed in real nonlinear systems. Since then, the existence of three-tori has been confirmed in experiments on electronic circuits [11, 12], solid mechanics [13], hydrodynamics [14], Rayleigh-Bénard convection [15] and magnetohydrodynamics (MHD) [16]. The study of MHD flows is of fundamental relevance in geophysics and astrophysics which motivated experiments [17-20] and simulations [21, 22] that investigate the role of chaotic and/or turbulent flows for planetary and stellar dynamos [23], or the turbulent transport processes occurring in accretion disks [24] where the magnetorotational instability (MRI) [25] plays a basic role.

Symmetries in physical systems provide a way to circumvent the NRT theorem because the bifurcations occurring in these systems may be non-generic. Their understanding is of relevance as the character of an underlying symmetry group in general is reflected in the possible solutions and their evolution in time, e.g. in terms of conservation laws. This is especially the case in fluid dynamics [26], or magnetohydrodynamics, where the appearance of threetori solutions has been interpreted as a consequence of bifurcations [16, 27], which may introduce a breaking of symmetry $[8,28,29]$. Quasiperiodic tori with more than three frequencies are however rarely found in systems with moderate and large number of degrees of freedom (e.g. [6]). For instance, the existence of four-tori solutions has been attributed to the non-generic character of two-dimensional Rayleigh-Bénard convection [30], or to spatial localisation of weakly coupled individual modes in $[15,31]$. The latter studies pointed out the relevance of the spatial structure of the solutions for the emergence of chaos in large-scale systems.

In this Letter we investigate the emergence of four-tori and chaotic flows in simulations of a magnetised spherical Couette (MSC) system. Using an accurate frequency analysis based on Laskar's algorithm [32] and Poincaré sections, we find that consecutive symmetry breaking caused by various Hopf bifurcations determine the evolution of the system and accompany the route to chaotic behaviour. The MSC system constitutes a paradigmatic MHD problem [33-36] that is of relevance for differentially rotating, electrically conducting flows. Flows driven by differential rotation, which have been investigated in several experiments [37-42], govern the dynamics in the interior of stars and/or planets [22] where they constitute a possible source for MHD wave phenomena [43], dynamo action [44], and perhaps even for the generation of gravitational wave signals from neutron stars [45, 46].

In terms of symmetry theory [47] the MSC problem is a $\mathbf{S O}(2) \times \mathbf{Z}_{2}$ equivariant system, i.e., invariant by azimuthal rotations $(\mathbf{S O}(2))$ and reflections with respect to the equatorial plane $\left(\mathbf{Z}_{2}\right)$. In $\mathbf{S O}(2)$ symmetric systems, branches of rotating waves (RWs), either stable or unstable, appear after the axisymmetric base state becomes unstable (primary Hopf bifurcation [26]). Successive Hopf bifurcations [48, 49] give rise to quasiperiodic modulated rotating waves (MRWs) and to chaotic turbulent flows, usually following the NRT scenario [48]. In the particular case of the MSC, when the magnetic field is varied, branches of RWs with a $m$-fold azimuthal symmetry with a prime number $m=2,3[34,50]$ give rise to stable twoand three-tori MRWs [51], and eventually chaotic flows [8], though four-tori MRWs have not yet been found. We show below that MHD four-tori solutions in terms of MRWs can be obtained after successive azimuthal symmetry breaking 
Hopf bifurcations from a parent branch of RW having $m$-fold symmetry which is not a prime number, in this case $m=4$. Note that when $m$ is a prime number only one symmetry breaking bifurcation can take place as the flows are equatorially asymmetric so that the case $m=4$ constitutes the lowest non-trivial possibility for azimuthal symmetry breaking with multiple bifurcations.

We consider an electrically conducting fluid of density $\rho$, kinematic viscosity $\nu$, magnetic diffusivity $\eta=1 /\left(\sigma \mu_{0}\right)$ (where $\mu_{0}$ is the magnetic permeability of the free-space and $\sigma$ is the electrical conductivity). The fluid is bounded by two spheres with radius $r_{\mathrm{i}}$ and $r_{\mathrm{o}}$, respectively, with the outer sphere being at rest and the inner sphere rotating at angular velocity $\Omega$ around the vertical axis $\hat{\mathbf{e}}_{\mathbf{z}}$ (see inset of Fig. 1). A uniform axial magnetic field of amplitude $B_{0}$ is imposed as in the HEDGEHOG experiment [40]. Scaling the length, time, velocity and magnetic field with $d=r_{\mathrm{o}}-r_{\mathrm{i}}, d^{2} / \nu, r_{\mathrm{i}} \Omega$ and $B_{0}$, respectively, the temporal evolution of the system is governed by the Navier-Stokes equation and the induction equation which read:

$$
\begin{aligned}
& \partial_{t} \mathbf{v}+\operatorname{Re}(\mathbf{v} \cdot \nabla) \mathbf{v}=-\nabla p+\nabla^{2} \mathbf{v}+\mathrm{Ha}^{2}(\nabla \times \mathbf{b}) \times \hat{\mathbf{e}}_{\mathbf{z}}, \\
& 0=\nabla \times\left(\mathbf{v} \times \hat{\mathbf{e}}_{\mathbf{z}}\right)+\nabla^{2} \mathbf{b}, \quad \nabla \cdot \mathbf{v}=0, \quad \nabla \cdot \mathbf{b}=0,
\end{aligned}
$$

where $\operatorname{Re}=\Omega r_{\mathrm{i}} d / \nu$ is the commonly known Reynolds number, $\mathrm{Ha}=B_{0} d(\sigma /(\rho \nu))^{1 / 2}$ is the Hartmann number, $\mathbf{v}$ the velocity field and $\mathbf{b}$ the deviation of magnetic field from the axial applied field. Here we use the inductionless approximation which is valid in the limit of small magnetic Reynolds number, $\mathrm{Rm}=\Omega r_{\mathrm{i}} d / \eta \ll 1$. This condition is well met when considering the liquid metal GaInSn, with magnetic Prandtl number $\mathrm{Pm}=\nu / \eta \sim O\left(10^{-6}\right)$ [52], at moderate $\mathrm{Re}=10^{3}$ (similar to the experiment [40]) since $\mathrm{Rm}=\operatorname{Pm} \operatorname{Re} \sim 10^{-3}$. The aspect ratio is $\chi=r_{\mathrm{i}} / r_{\mathrm{o}}=0.5$ and no-slip $\left(v_{r}=v_{\theta}=v_{\varphi}=0\right)$ at $r=r_{\mathrm{o}}$ and constant rotation $\left(v_{r}=v_{\theta}=0, v_{\varphi}=\sin \theta, \theta\right.$ being colatitude) at $r=r_{\mathrm{i}}$ are the boundary conditions imposed on the velocity field. For the magnetic field, insulating boundary conditions are considered in accordance with typical experimental setups $[37,53]$. Spectral methods -spherical harmonics in the angular coordinates and a collocation method in the radial direction- and high order implicit-explicit backward-differentiation (IMEX-BDF) time schemes are employed for solving the MSC equations (see $[8,50]$ for details).

The solutions are classified according to their azimuthal symmetry $m$, the wave number with the largest volumeaveraged kinetic energy $m_{\max }$, and their type of time dependence. In this way, branches of RWs and MRWs are labelled as $\mathrm{RW}_{m}^{m_{\max }}$ and $\mathrm{MRW}_{m}^{m_{\max }}$. The latter can be quasiperiodic with 2, 3 and 4 frequencies and are labelled as $2 \mathrm{~T}, 3 \mathrm{~T}$ and $4 \mathrm{~T}$, respectively. The branches of equatorially asymmetric $\mathrm{RW}_{2}^{2}, \mathrm{RW}_{3}^{3}$, and $\mathrm{RW}_{4}^{4}$ which bifurcate from the base state at $\mathrm{Ha}=12.2[34]$ were computed in [50] by means of continuation methods [54-56]. The latter allows, for each parameter, to find a periodic solu-

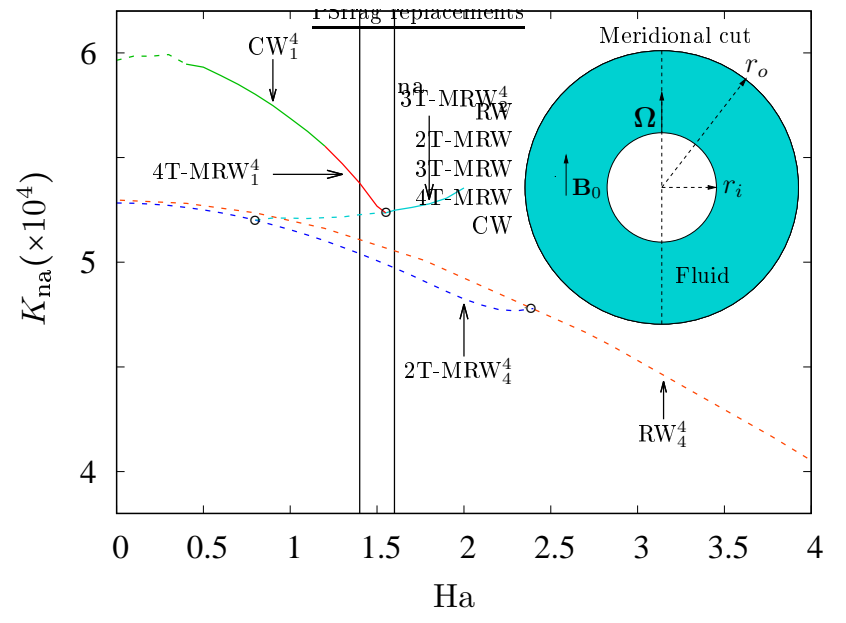

FIG. 1. Magnetised spherical Couette (MSC) geometry and bifurcation diagram of the volume-averaged nonaxisymmetric kinetic energy density $K_{\text {na }}$ versus Ha. Solid (dashed) lines are used for stable (unstable) flows. Branches of rotating waves $\mathrm{RW}_{m}^{m_{\max }}$, modulated rotating waves $\mathrm{MRW}_{m}^{m_{\max }}$, and complex waves $\mathrm{CW}_{m}^{m_{\max }}$ are shown. MRWs with 2, 3 and 4 frequencies are labelled as $2 \mathrm{~T}, 3 \mathrm{~T}$ and $4 \mathrm{~T}$, respectively. The colours distinguish the various solutions according to the corresponding label. The vertical lines mark Ha for the solutions selected for Fig. 2.

tion by applying a Newton method to a function derived from the flow periodicity condition (see [56] for a detailed description). Here we focus on the analysis of MRW bifurcating from the unstable branch $\mathrm{RW}_{4}^{4}$ for a small control parameter $\mathrm{Ha}<2.5$ and fixed $\mathrm{Re}=10^{3}$. These MRWs have been successively obtained by means of direct numerical simulations (DNS) of the MSC equations with $n_{r}=40$ radial collocation points and a spherical harmonic truncation parameter of $L_{\max }=84$. The dimension of the system is then $n=\left(2 L_{\max }^{2}+4 L_{\max }\right)\left(n_{r}-1\right)=563472$. The results for the four-tori solution at $\mathrm{Ha}=1.4$ are confirmed for increased resolution with $n_{r}=60$ and $L_{\max }=126$. Azimuthal symmetry $m=m_{d}$ can be imposed on the DNS by only considering the azimuthal wave numbers $m=k m_{d}, k \in \mathbb{Z}$ in the spherical harmonic expansion of the fields. All DNS comprise more than 100 viscous time units and initial transients less than 10 time units are required before the statistically saturated state is reached. The time and volume-averaged nonaxisymmetric kinetic energy density $K_{\text {na }}$ is employed as a proxy of the time dependence of the flows because they initially bifurcate from an axisymmetric base state (only the $m=0$ mode is nonzero). For each Ha a new MRW is computed from a previous state with nearby Ha. We usually use $\Delta \mathrm{Ha}=0.1$, but smaller values are selected close to a bifurcation. The first branch of MRW which we compute here, bifurcates from the unstable branch $\mathrm{RW}_{4}^{4}$, already computed in [50].

The bifurcation diagram in Fig. 1 displays $K_{\text {na }}$ versus $\mathrm{Ha}$ and the bifurcation points are marked with circles. By 

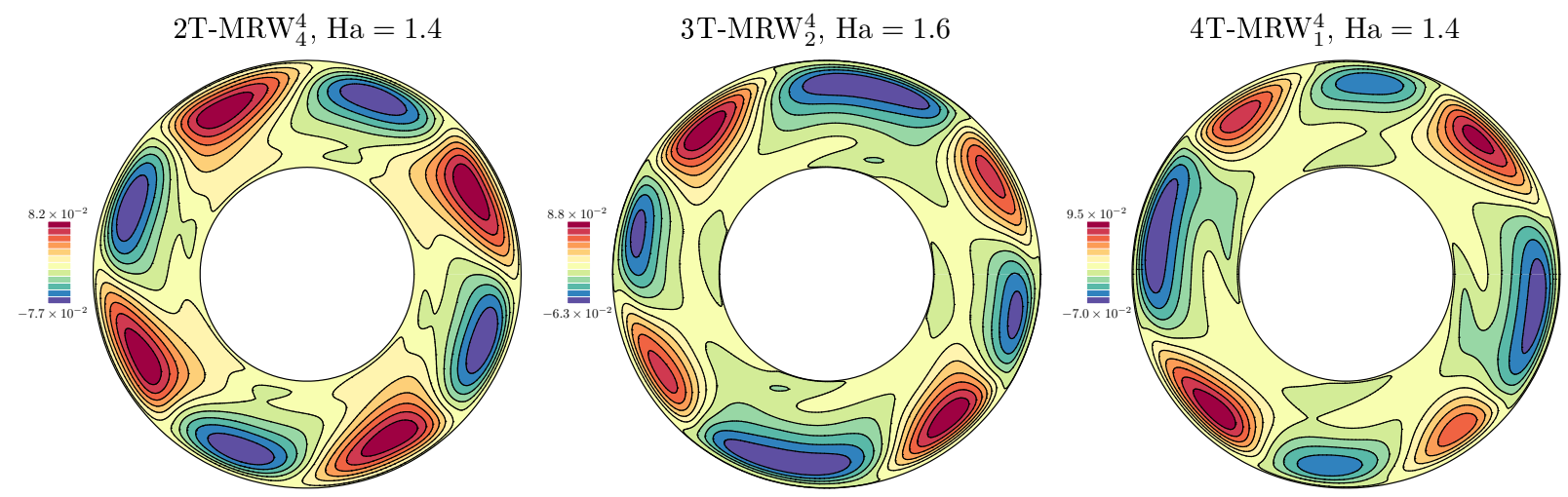

FIG. 2. Contour plots of the nonaxisymmetric component of the radial velocity on a colatitudinal section at $\theta \approx 93^{\circ}$. The azimuthal symmetry is broken from left to right: from $m=4$ to $m=2$ and from $m=2$ to $m=1$ (compare the shape of the opposite red cells).
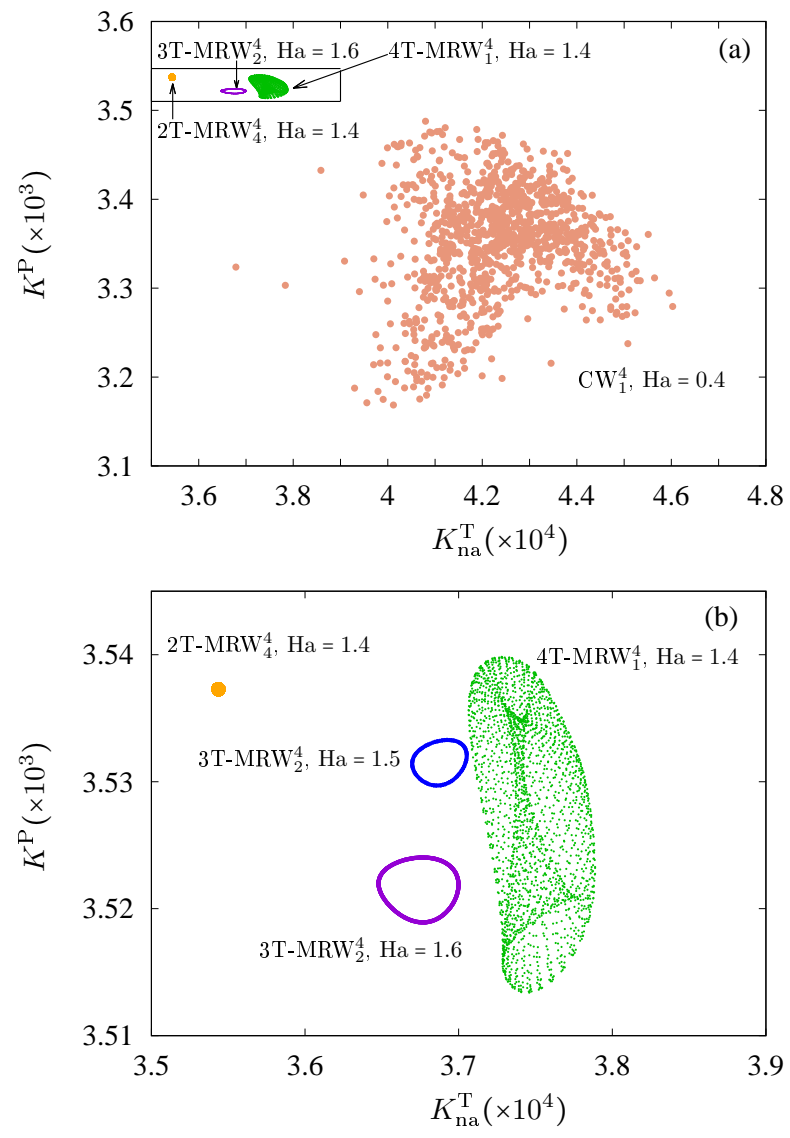

FIG. 3. Poincaré sections at the time instants $t_{i}$ defined by the constraint $K\left(t_{i}\right)=\bar{K}, K$ being the volume-averaged kinetic energy and $\bar{K}$ its time average. The volume-averaged poloidal kinetic energy $K^{\mathrm{P}}\left(t_{i}\right)$ is displayed versus the volume-averaged toroidal nonaxisymmetric energy $K_{\mathrm{na}}^{\mathrm{T}}\left(t_{i}\right)$. (a) $2 \mathrm{~T}-\mathrm{MRW}_{4}^{4}$ at $\mathrm{Ha}=1.4,3 \mathrm{~T}-\mathrm{MRW}_{2}^{4}$ at $\mathrm{Ha}=1.6,4 \mathrm{~T}-\mathrm{MRW}_{1}^{4}$ at $\mathrm{Ha}=1.4$, and a chaotic wave $\mathrm{CW}_{1}^{4}$ at $\mathrm{Ha}=1.4$. (b) Detail of (a) showing also a $3 \mathrm{~T}-\mathrm{MRW}_{2}^{4}$ at $\mathrm{Ha}=1.5$. decreasing Ha, periodic $\mathrm{RW}_{4}^{4}$ undergo a Hopf bifurcation to $2 \mathrm{~T}-\mathrm{MRW}_{4}^{4}$ around $\mathrm{Ha} \approx 2.4$ which then extends down to $\mathrm{Ha}=0$. These flows are obtained with time integrations with the azimuthal symmetry constrained to $m=4$ and are unstable to small random perturbations with azimuthal symmetry $m=1$. Another branch of unstable solutions appears at $\mathrm{Ha}=0.8$ via a secondary subcritical Hopf bifurcation on the $2 \mathrm{~T}-\mathrm{MRW}_{4}^{4}$ branch (blue dashed curve) which breaks the $m=4$ symmetry to $m=2$ and finally leads to the emergence of an unstable $3 \mathrm{~T}-\mathrm{MRW}_{2}^{4}$ branch (light blue dashed curve). The latter extends for increasing $\mathrm{Ha}$ and becomes stable at $\mathrm{Ha} \approx 1.52$ where a branch of $4 \mathrm{~T}-\mathrm{MRW}_{1}^{4}$ is born thanks to a tertiary subcritical Hopf bifurcation breaking the $m=2$ symmetry (red curve). This branch is lost for Ha $\lesssim 1.35$ (green curve) and complex flows, either with more than four frequencies or chaotic, occur. The contour plots of the $m \neq 0 \mathrm{com}-$ ponent of the radial velocity, on a colatitudinal section slightly below the equatorial plane, are displayed in Fig. 2 for one example of each type of MRW with azimuthal symmetry $m=4, m=2$, and $m=1$ (from left to right).

The time dependence of RWs is described by a uniform azimuthal rotation of a fixed flow pattern whereas for MRWs the pattern is azimuthally rotating but modulated with additional frequencies (e.g. [48]). Thus, a frequency analysis of any azimuthally averaged property provides one frequency less than the analysis of a localised particular flow component. Because of this, Poincaré sections at the time instants $t_{i}$ defined by the constraint $K\left(t_{i}\right)=\bar{K}$, $K$ being the volume-averaged kinetic energy and $\bar{K}$ its time average, appear as a single point for $2 \mathrm{~T}$, a closed curve for $3 \mathrm{~T}$, a band of points for $4 \mathrm{~T}$, and a cloud of points for chaotic flows (Fig. 3). To confirm the regular behavior of 2T, 3T, and 4T MRWs we perform a frequency analysis. The frequencies giving rise to the modulation are accurately determined from the time series of $K_{4}(K$ restricted to the $m=4$ mode) by means of a Fourier transform based optimisation algorithm by Laskar [32]. If the 
solution is regular the frequencies do not depend (within the frequency determination accuracy) on the particular time window used for the analysis $[57,58]$. Sufficiently wide time windows $(5-40$ time units) over large time series (100 time units) are considered which leads to a relative accuracy around $10^{-5}$.

The power spectral density (psd) for $K_{4}$ is displayed in Fig. 4 for the same MRW as shown in Fig. 2 additionally including the psd for a chaotic solution at $\mathrm{Ha}=0.4$ (bottom curve). Some examples for the fundamental frequencies or corresponding linear combinations $\sum k_{i} f_{i}$ with $k_{i}$ being integers, are explicitly marked. In all cases we have checked that the relative accuracy $\left|f-\sum k_{i} f_{i}\right| / f<10^{-5}$, with $-6 \leq k_{i} \leq 6$, for all frequencies obtained with Laskar's algorithm. In the simplest case, the $2 \mathrm{~T}-\mathrm{MRW}_{4}^{4}$ at $\mathrm{Ha}=1.4$, only one fundamental frequency $f_{1}$ and its multiples are present, because the fundamental frequency associated to the drift motion is removed by volume averaging. A second frequency $f_{2}$ and few combinations $k_{1} f_{1}+k_{2} f_{2}$ occur for the $3 \mathrm{~T}-\mathrm{MRW}_{2}^{4}$ whereas the psd of $4 \mathrm{~T}-\mathrm{MRW}_{1}^{4}$ reveals a complex time dependence with several combinations of the type $k_{1} f_{1}+k_{2} f_{2}+k_{3} f_{3}$. For $\mathrm{Ha} \lesssim 1.35$ the variation of the main frequency $f_{1}$ becomes larger than the accuracy and thus $4 \mathrm{~T}-\mathrm{MRW}_{1}^{4}$ give rise to more complex motions, which could be regular flows with a very small additional frequency or chaotic flows. However, deciding whether a new very small frequency has appeared in this regime would require extremely long time integrations of the MSC system, which are out of the scope of the present study. Nevertheless, it is clear that fully broadband frequency distribution characteristic for chaotic flows is obtained for $\mathrm{Ha} \lesssim 0.7$. The psd for the chaotic solution at $\mathrm{Ha}=0.4$ shown in Fig. 4 (bottom curve) exhibits a noticeable peak that corresponds to the main frequency of modulation of the original MRW.

As suggested in [15], the strong polar localisation of the $m=1$ (also $m=3$ ) perturbations (see Fig. 1, supplementary material) provides a way to overcome the NRT requirements and thus explains the existence of $4 \mathrm{~T}-\mathrm{MRW}_{1}^{4}$. In our case, successive azimuthal symmetry breaking bifurcations from unstable regular states (that can not be realised in the experiments of [15]) are responsible for the mode localisation. For the regular solutions, the kinetic energy fluctuations of the different modes evidence a weak nonlinear interaction, being the $m=4$ component which most contributes to the flow (see Figure 2 supplementary material, also the kinetic energy spectra in Fig. 5). The amplitude of fluctuations leading to chaotic flows seem not to be associated to turbulent spatial behaviour as the $m=4$ component of the flow still dominates over the other modes (Fig. 5).

The present study evidences that four-tori solutions are physically possible in MHD problems and can be explained in terms of bifurcation theory. The bifurcation scenario resembles the NRT scenario but involves two additional Hopf bifurcations, including a subcritical bifurcation lead-

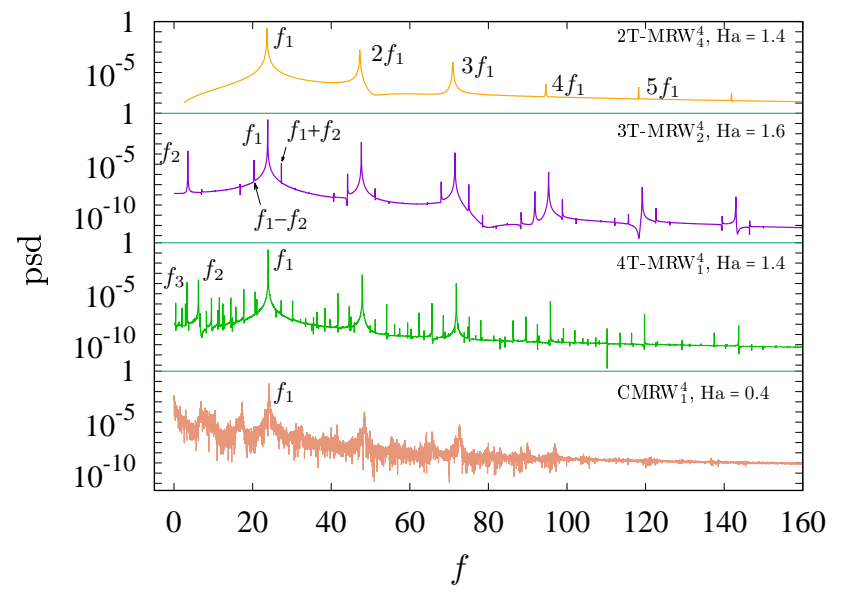

FIG. 4. Power spectral density for the volume-averaged kinetic energy density $K_{4}$ of the $m=4$ mode for $2 \mathrm{~T}-\mathrm{MRW}_{4}^{4}$ at $\mathrm{Ha}=1.4,3 \mathrm{~T}-\mathrm{MRW}_{2}^{4}$ at $\mathrm{Ha}=1.6,4 \mathrm{~T}-\mathrm{MRW}_{1}^{4}$ at $\mathrm{Ha}=1.4$, and a chaotic wave $\mathrm{CW}_{1}^{4}$ at $\mathrm{Ha}=1.4$. The time series of $K_{4}$ are displayed on Fig. 2 of the supplementary material.

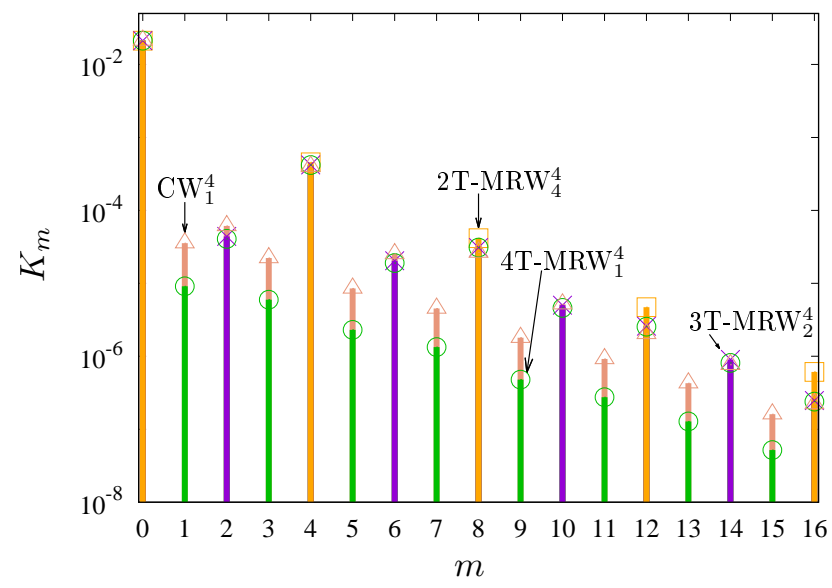

FIG. 5. (a) Kinetic energy spectra $\left(K_{m}\right.$ versus $m$ ) for $2 \mathrm{~T}$ $\mathrm{MRW}_{4}^{4}$ at $\mathrm{Ha}=1.4(\square), 3 \mathrm{~T}-\mathrm{MRW}_{2}^{4}$ at $\mathrm{Ha}=1.6(\times), 4 \mathrm{~T}-$ $\mathrm{MRW}_{1}^{4}$ at $\mathrm{Ha}=1.4(\mathrm{O})$, and a chaotic flow $\mathrm{CW}_{1}^{4}$ at $\mathrm{Ha}=0.4$ $(\triangle)$.

ing to a stabilisation of a three-tori solution. This kind of stabilisation was also found in [59], but for axisymmetric steady states, arising in purely hydrodynamic spherical Couette (SC) flows. Further SC experiments [60] are in accordance with the NRT scenario, but only two-tori were detected before the regime of chaotic flows. Similarly, three or four-tori have not been found in a comprehensive study of the different flow regimes in the SC system with positive or negative differential rotation [36]. When the magnetic field is included, several MSC regimes have been studied recently [33, 41, 61] but the existence of quasiperiodic flows with three frequencies has not been shown until [8].

The fundamental result presented here is that in a system with symmetry, more symmetry breaking bifurcations 
may be required in the NRT scenario until a flow can become chaotic, and thus regular motions with three or four frequencies are likely to occur, formed by modes localised in different parts of the domain [15]. These flows originate from unstable states of azimuthal symmetry $m=4$ and thus their origin can only be understood with symmetrically constrained simulations and not with experiments. Speculatively, periodic flows with an azimuthal symmetry that constitute a product of several prime numbers, may give rise to quasiperiodic flows with more than four frequencies, as several symmetry breaking bifurcations can occur. The new frequency occurring at the bifurcation is usually smaller (but also can be larger, e.g. [15]) than the previous fundamental one, as can be seen in Fig. 4. With on going bifurcations the effect requires a longer time evolution of the system until the upcoming frequency can be detected in the captured time series. The smallest fundamental frequency of the simulated $4 \mathrm{~T}-\mathrm{MRW}_{1}^{4}$ at $\mathrm{Ha}=1.4$ gives rise to a time scale of around 1600 seconds which should be detectable in the HEDGEHOG experiment [40], whose duration is limited to 10 hours due to the decrease of signal quality of flow measurement [53]. The results also bear relevance for the MRI as previous experiments [37] may be understood in terms of MSC instabilities [33, 61], similar to those analysed here.

\section{SUPPLEMENTARY MATERIAL}

The modulated rotating waves (MRWs) presented in the main manuscript have been successively obtained by means of direct numerical simulations (DNS) of the magnetised spherical Couette (MSC) equations with $n_{r}=40$ radial collocation points and a spherical harmonic truncation parameter of $L_{\max }=84$. The dimension of the system is then $n=\left(2 L_{\max }^{2}+4 L_{\max }\right)\left(n_{r}-1\right)=563472$. The results for the four-tori solution at $\mathrm{Ha}=1.4$ are confirmed for increased resolution with $n_{r}=60$ and $L_{\max }=126$. Azimuthal symmetry $m=m_{d}$ can be imposed on the DNS by only considering the azimuthal wave numbers $m=k m_{d}, k \in \mathbb{Z}$ in the spherical harmonic expansion of the fields. All DNS comprise more than 100 viscous time units and initial transients less than 10 time units are required before the statistically saturated state is reached. For each Ha a new MRW is computed from a previous state with nearby $\mathrm{Ha}$. We usually use $\Delta \mathrm{Ha}=0.1$, but smaller values are selected close to a bifurcation. The first branch of MRW which we compute in the present study, bifurcates from the unstable branch $\mathrm{RW}_{4}^{4}$, already computed in [50].

Figure 6 corresponds to the $4 \mathrm{~T}-\mathrm{MRW}_{1}^{4}$ at $\mathrm{Ha}=1.4$ and displays, from left to right and from top to bottom, the contour plots of the kinetic energy density, on a meridional section through a relative maxima, for the $m=1, m=2$, $m=3$ and $m=4$ modes, respectively. While fluid motions remain confined to mid and low latitudes in the
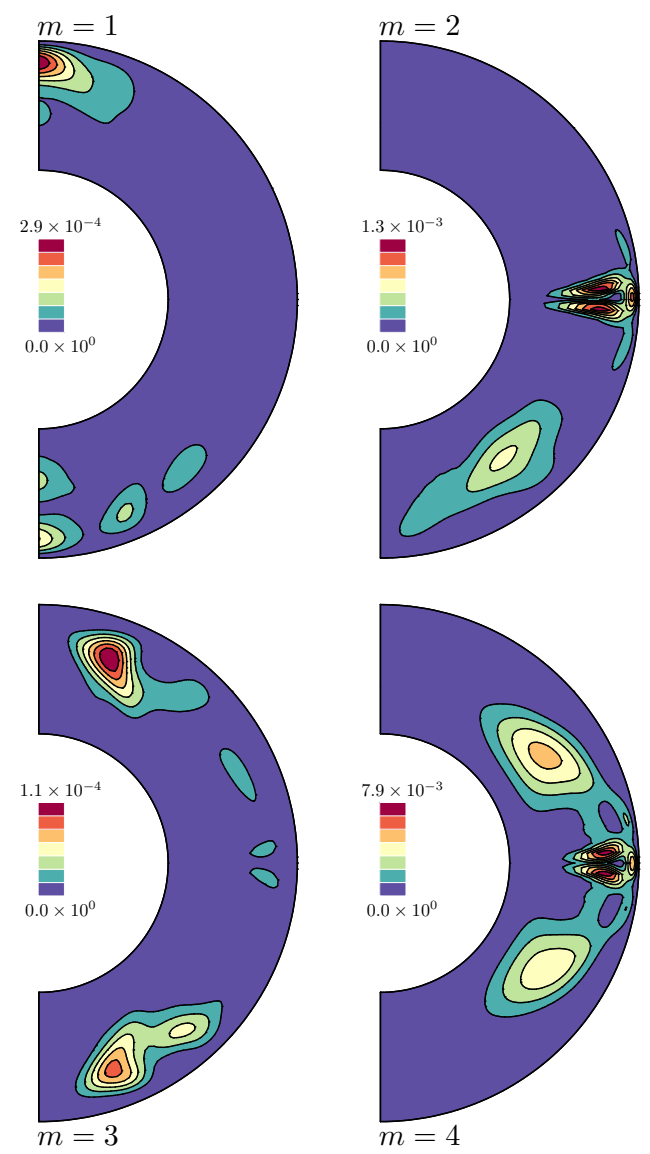

FIG. 6. Contour plots of the $m=1,2,3,4$ components (from left to right and from top to bottom) of the kinetic energy density on a meridional section through a relative maximum. The solution is the $4 \mathrm{~T}-\mathrm{MRW}_{1}^{4}$ at $\mathrm{Ha}=1.4$ displayed on the rightmost plot of Fig. 2 of the main manuscript.

case of $m=2$ and $m=4$ modes (even modes), the flow is restricted to high latitudes in the case of odd modes $(m=1$ and $m=3)$. Then, even modes contribute to motions outside the tangent cylinder (an imaginary cylinder parallel to the rotation axis and tangent to the inner sphere) whereas odd modes contribute to motions within the tangent cylinder. As suggested in [15] the different localisation of the different modes within the domain may explain the appearance of flows with four fundamental frequencies.

To describe the nature of kinetic energy fluctuations, the time series of the volume-averaged kinetic energy of each azimuthal wave number $m=1,2,3,4$ is displayed in figure $7(\mathrm{a}-\mathrm{c})$ for the three types of MRW shown in Figs. 2,4 and 5 of the main manuscript: A $2 \mathrm{~T}-\mathrm{MRW}_{4}^{4}$, a $3 \mathrm{~T}-$ $\mathrm{MRW}_{2}^{4}$, and a $4 \mathrm{~T}-\mathrm{MRW}_{1}^{4}$. The dominance of the $m=4$ component of the flow and the quasiperiodic character of the waves are clear from the figure. The modes arising at the symmetry breaking bifurcations $(m=1,2,3)$ have larger fluctuations than the main $m=4$ component of the flow, but barely contribute to the total kinetic energy. 

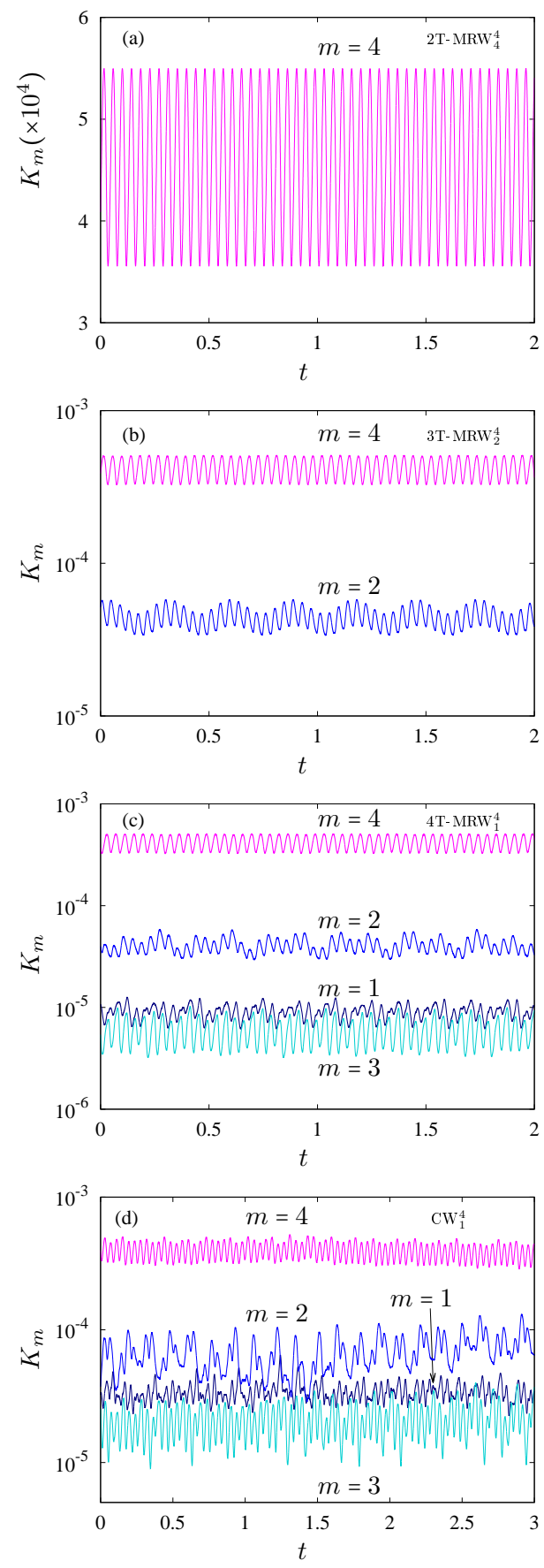

FIG. 7. Time series of the volume-averaged kinetic energy contained in each azimuthal wave number $m=1,2,3,4$. (a) Two frequency solution $2 \mathrm{~T}-\mathrm{MRW}_{4}^{4}$ at $\mathrm{Ha}=1.4$. Only the modes $m=4 k, k \in \mathbb{Z}$ are nonzero. (b) Three frequency solution $3 \mathrm{~T}-\mathrm{MRW}_{2}^{4}$ at $\mathrm{Ha}=1.6$. Only the modes $m=2 k, k \in \mathbb{Z}$ are nonzero. (c) Four frequency solution $4 \mathrm{~T}-\mathrm{MRW}_{1}^{4}$ at $\mathrm{Ha}=1.4$. All the modes $m \in \mathbb{Z}$ are nonzero. (d) Chaotic solution at $\mathrm{Ha}=0.4$. Panels (a),(b) and (c) correspond to the contour plots, from left to right respectively, shown in figure 2 of the main manuscript. The power spectral density of the time series corresponding to $K_{4}$ are displayed on figure 4 of the main manuscript.
The time series for a chaotic solution $\mathrm{CW}_{1}^{4}$ is plotted in Fig. 7(d) and shows a larger contribution of the nondominant $m=1,2,3$ modes.

F. G. was supported by the Alexander von Humboldt Foundation. This project has also received funding from the European Research Council (ERC) under the European Union's Horizon 2020 research and innovation programme (grant agreement No 787544).

[1] G. B. Ermentrout, "The behavior of rings of coupled oscillators," J. Math. Biol. 23, 55-74 (1985).

[2] M. G. Grigorov, "Global dynamics of biological systems from time-resolved omics experiments," Bioinformatics 22, 1424-1430 (2006).

[3] H.-W. Lorenz, "Strange attractors in a multisector business cycle model," J. Econ. Behav. Organ. 8, 397 - 411 (1987).

[4] L. A. Safonov, E. Tomer, V. V. Strygin, Y. Ashkenazy, and S. Havlin, "Multifractal chaotic attractors in a system of delay-differential equations modeling road traffic," CHAOS 12, 1006-1014 (2002).

[5] O. Hess, D. Merbach, H.-P. Herzel, and E. Scholl, "Bifurcations of a three-torus in a twin-stripe semiconductor laser model," Phys. Lett. A 194, 289 - 294 (1994).

[6] D.J. Albers and J.C. Sprott, "Routes to chaos in highdimensional dynamical systems: A qualitative numerical study," Physica D 223, 194-207 (2006).

[7] D. Castaño, M. C. Navarro, and H. Herrero, "Evolution of secondary whirls in thermoconvective vortices: Strengthening, weakening, and disappearance in the route to chaos," Phys. Rev. E 93, 013117 (2016).

[8] F. Garcia, M. Seilmayer, A. Giesecke, and F. Stefani, "Chaotic wave dynamics in weakly magnetised spherical Couette flows," CHAOS 30, 043116 (2020).

[9] S. Newhouse, D. Ruelle, and F. Takens, "Occurrence of strange axiom A attractors near quasiperiodic flows on $t^{m}, m \geq 3$," Commun. Math. Phys. 64, 35-40 (1978).

[10] C. Grebogi, E. Ott, and J. A. Yorke, "Are three-frequency quasiperiodic orbits to be expected in typical nonlinear dynamical systems?" Phys. Rev. Lett. 51, 339-342 (1983).

[11] A. Cumming and P. S. Linsay, "Quasiperiodicity and chaos in a system with three competing frequencies," Phys. Rev. Lett. 60, 2719-2722 (1988).

[12] E. Sánchez, D. Pazó, and M. A. Matías, "Experimental study of the transitions between synchronous chaos and a periodic rotating wave," CHAOS 16, 033122 (2006).

[13] R. Alaggio and G. Rega, "Characterizing bifurcations and classes of motion in the transition to chaos through 3D-tori of a continuous experimental system in solid mechanics," Physica D 137, 70-93 (2000).

[14] J. Langenberg, G. Pfister, and J. Abshagen, "Chaos from Hopf bifurcation in a fluid flow experiment," Phys. Rev. E 70, 046209 (2004).

[15] R. W. Walden, P. Kolodner, A. Passner, and C. M. Surko, "Nonchaotic rayleigh-bénard convection with four and five incommensurate frequencies," Phys. Rev. Lett. 53, 242-245 (1984).

[16] A. Libchaber, S. Fauvé, and C. Laroche, "Two-parameter study of the routes to chaos," Physica D 7, 73 - 84 (1983). 
[17] F. Stefani, T. Gundrum, G. Gerbeth, G. Rüdiger, M. Schultz, J. Szklarski, and R. Hollerbach, "Experimental evidence for magnetorotational instability in a Taylor-Couette flow under the influence of a helical magnetic field," Phys. Rev. Lett. 97, 184502 (2006).

[18] A. Gailitis, O. Lielausis, E. Platacis, G Gerbeth, and F. Stefani, "Colloquium: Laboratory experiments on hydromagnetic dynamos," Rev. Modern Phys. 74, 973-990 (2002).

[19] B. Gallet, S. Aumaître, J. Boisson, F. Daviaud, B. Dubrulle, N. Bonnefoy, M. Bourgoin, Ph. Odier, J.-F. Pinton, N. Plihon, G. Verhille, S. Fauve, and F. Pétrélis, "Experimental observation of spatially localized dynamo magnetic fields," Phys. Rev. Lett. 108, 144501 (2012).

[20] Z. Stelzer, S. Miralles, D. Cébron, J. Noir, S. Vantieghem, and A. Jackson, "Experimental and numerical study of electrically driven magnetohydrodynamic flow in a modified cylindrical annulus. II. Instabilities," Phys. Fluids 27, 084108 (2015).

[21] P. H. Roberts and G. A. Glatzmaier, "Geodynamo theory and simulations," Rev. Modern Phys. 72, 1081-1123 (2000).

[22] C. A. Jones, "Planetary magnetic fields and fluid dynamos," Ann. Rev. Fluid Mech. 43, 583-614 (2011).

[23] P. H. Roberts and M. Stix, "The turbulent dynamo: A translation of a series of papers by F. Krause, K.-H Radler, and M. Steenbeck," NCAR Technical Notes Collection TN-60+IA (1971).

[24] S. A. Balbus and J. F. Hawley, "Instability, turbulence, and enhanced transport in accretion disks," Rev. Modern Phys. 70, 1-53 (1998).

[25] S. A. Balbus and J. F. Hawley, "A powerful local shear instability in weakly magnetized disks. i- Linear analysis. ii- Nonlinear evolution," Astrophys. J. 376, 214-233 (1991).

[26] J. D. Crawford and E. Knobloch, "Symmetry and symmetry-breaking bifurcations in fluid dynamics," Ann. Rev. Fluid Mech. 23, 341-387 (1991).

[27] J. M. Lopez and F. Marques, "Dynamics of three-tori in a periodically forced Navier-Stokes flow," Phys. Rev. Lett. 85, 972-975 (2000).

[28] S. Altmeyer, Y. Do, F. Marques, and J. M. Lopez, "Symmetry-breaking Hopf bifurcations to 1-, 2-, and 3tori in small-aspect-ratio counterrotating Taylor-Couette flow," Phys. Rev. E 86, 046316 (2012).

[29] F. Garcia, M. Net, and J. Sánchez, "Continuation and stability of convective modulated rotating waves in spherical shells," Phys. Rev. E 93, 013119 (2016).

[30] E. Zienicke, N. Seehafer, and F. Feudel, "Bifurcations in two-dimensional Rayleigh-Bénard convection," Phys. Rev. E 57, 428-435 (1998).

[31] K. He, "Riddling of the orbit in a high dimensional torus and intermittent energy bursts in a nonlinear wave system," Phys. Rev. Lett. 94, 034101 (2005).

[32] J. Laskar, "Frequency analysis of a dynamical system," Celestial Mech. Dyn. Astr. 56, 191-196 (1993).

[33] R. Hollerbach, "Non-axisymmetric instabilities in magnetic spherical Couette flow," Proc. Roy. Soc. Lond. A 465, 2003-2013 (2009).

[34] V. Travnikov, K. Eckert, and S. Odenbach, "Influence of an axial magnetic field on the stability of spherical Couette flows with different gap widths," Acta Mech. 219, 255-268 (2011).

[35] A. Figueroa, N. Schaeffer, H.-C. Nataf, and D. Schmitt,
"Modes and instabilities in magnetized spherical Couette flow," J. Fluid Mech. 716, 445-469 (2013).

[36] Johannes Wicht, "Flow instabilities in the wide-gap spherical Couette system," J. Fluid Mech. 738, 184-221 (2014).

[37] D. R. Sisan, N. Mujica, W. A. Tillotson, Y. M. Huang, W. Dorland, A. B. Hassam, T. M. Antonsen, and D. P. Lathrop, "Experimental observation and characterization of the magnetorotational instability," Phys. Rev. Lett. 93, 114502 (2004).

[38] D. S. Zimmerman, S. A. Triana, and D. P. Lathrop, "Bi-stability in turbulent, rotating spherical Couette flow," Phys. Fluids 23, 065104 (2011).

[39] M. Hoff, U. Harlander, and C. Egbers, "Experimental survey of linear and nonlinear inertial waves and wave instabilities in a spherical shell," J. Fluid Mech. 789, 589616 (2016).

[40] C. Kasprzyk, E. Kaplan, M. Seilmayer, and F. Stefani, "Transitions in a magnetized quasi-laminar spherical Couette flow," Magnetohydrodynamics 53, 393-401 (2017).

[41] E. J. Kaplan, H.-C. Nataf, and N. Schaeffer, "Dynamic domains of the Derviche Tourneur sodium experiment: Simulations of a spherical magnetized Couette flow," Phys. Rev. Fluids 3, 034608 (2018).

[42] A. Barik, S. A. Triana, M. Hoff, and J. Wicht, "Triadic resonances in the wide-gap spherical Couette system," J. Fluid Mech. 843, 211-243 (2018).

[43] H. C. Spruit, "Dynamo action by differential rotation in a stably stratified stellar interior," Astron. \& Astrophys. 381, 923-932 (2002).

[44] A differentially rotating flow, for example, provides a natural explanation for the strong axisymmetric character of Saturn's magnetic field [62].

[45] C. Peralta, A. Melatos, M. Giacobello, and A. Ooi, "Gravitational radiation from nonaxisymmetric spherical Couette flow in a neutron star," Astrophys. J. Lett. 644, L53-L56 (2006).

[46] P. D. Lasky, "Gravitational Waves from Neutron Stars: A Review," Publications of the Astronomical Society of Australia 32, e034 (2015).

[47] M. Golubitsky and I. Stewart, The symmetry perspective: From equilibrium to chaos in phase space and physical space. (Birkhäuser, Basel, 2003).

[48] D. Rand, "Dynamics and symmetry. Predictions for modulated waves in rotating fluids," Arch. Ration. Mech. An. 79, 1-37 (1982).

[49] M. Golubitsky, V. G. LeBlanc, and I. Melbourne, "Hopf bifurcation from rotating waves and patterns in physical space," J. Nonlinear Sci. 10, 69-101 (2000).

[50] F. Garcia and F. Stefani, "Continuation and stability of rotating waves in the magnetized spherical Couette system: Secondary transitions and multistability," Proc. Roy. Soc. Lond. A 474, 20180281 (2018).

[51] F. Garcia, M. Seilmayer, A. Giesecke, and F. Stefani, "Modulated rotating waves in the magnetized spherical Couette system," J. Nonlinear Sci. 29, 2735-2759 (2019).

[52] Y. Plevachuk, V. Sklyarchuk, S. Eckert, G. Gerbeth, and R. Novakovic, "Thermophysical properties of the liquid Ga-In-Sn eutectic alloy," J. Chem. Eng. Data 59, 757-763 (2014)

[53] J. Ogbonna, F. Garcia, T. Gundrum, M. Seilmayer, and F. Stefani, "Experimental investigation of the return flow instability in magnetic spherical Couette flow," (2020), submitted to Phys. Fluids., arXiv:2009.07003 [physics.fludyn]. 
[54] E. Doedel and L. S. Tuckerman, eds., Numerical Methods for Bifurcation Problems and Large-Scale Dynamical Systems, IMA Volumes in Mathematics and its Applications, Vol. 119 (Springer-Verlag, Berlin, 2000).

[55] H. A. Dijkstra, F. W. Wubs, A. K. Cliffe, E. Doedel, I. F. Dragomirescu, B. Eckhardt, A. Y. Gelfat, A. L. Hazel, V. Lucarini, A. G. Salinger, E. T. Phipps, J. SánchezUmbría, H. Schuttelaars, L. S. Tuckerman, and U. Thiele, "Numerical bifurcation methods and their application to fluid dynamics: Analysis beyond simulation," Commun. Comput. Phys. 15, 1-45 (2014).

[56] J. Sánchez and M. Net, "Numerical continuation methods for large-scale dissipative dynamical systems," Eur. Phys. J. Spec. Top. 225, 2465-2486 (2016).

[57] J. Laskar, C. Froeschlé, and A. Celletti, "The measure of chaos by the numerical analysis of the fundamental fre- quencies. application to the standard mapping," Physica D 56, $253-269$ (1992).

[58] J. Laskar, "Frequency analysis for multi-dimensional systems. Global dynamics and diffusion," Physica D 67, 257-281 (1993).

[59] C. K. Mamun and L. S. Tuckerman, "Asymmetry and Hopf bifurcation in spherical Couette flow," Phys. Fluids 7, 80-91 (1995).

[60] P. Wulf, C. Egbers, and H. J. Rath, "Routes to chaos in wide-gap spherical Couette flow," Phys. Fluids 11, 1359-1372 (1999).

[61] C. Gissinger, H. Ji, and J. Goodman, "Instabilities in magnetized spherical Couette flow," Phys. Rev. E 84, 026308 (2011).

[62] J. Wicht and P. Olson, "Differential rotation dynamos: An application to Saturn," in Geophysical Research Abstracts (EGU General Assembly, 2010) p. 10179. 